\title{
Research on the Fitting Algorithm of GLONASS Broadcast Ephemeris Parameters
}

\author{
XIE Xiao Gang1, a, LU Ming Quan ${ }^{1, b^{*}}$ \\ ${ }^{1}$ Department of Electronic Engineering, Tsinghua University, Beijing, China \\ ajasonxx@163.com, blumq@tsinghua.edu.cn
}

\begin{abstract}
Keywords: Satellite Navigation System, GLONASS, Broadcast Ephemeris Parameters, Difference Quotient Approximation, User Range Error

Abstract. The accumulated error of GLONASS broadcast ephemeris is nearly 1 meters within 15 minutes due to simplified perturbation force model. Aiming at this problem, a new method of GLONASS broadcast ephemeris fitting algorithm is introduced. In this method, a nonlinear measurement model is built based on the GLONASS broadcast ephemeris user algorithm and linearized. And the partial derivative of Jacobin matrix is computed by difference quotient approximation. Then the GLONASS broadcast ephemeris parameters are estimated by using least square method. The simulation results show that the average user range error (URE) caused by fitting broadcast ephemeris is improved about two orders compared with the traditional broadcast ephemeris and achieves millimeter level. Finally, a verification system was built by the Japanese RTKLIB tools, the availability of the fitting broadcast ephemeris is validated from the positioning angle.
\end{abstract}

\section{Introduction}

The global navigation satellite system (GLONASS) of the former Soviet Union is equivalent to the global positioning system (GPS) of the United States. It has been built in early 1996 and put into operation. GLONASS is basically same as GPS in satellite constellation design and navigation positioning principle. But there are obvious differences in design of broadcast ephemeris parameters algorithm. The broadcast ephemeris parameters of GPS are based on the six Keplerian orbit elements, including the orbit perturbation harmonic coefficient and the time-dependent perturbation. They are extrapolated and forecasted every one or two hours by master station. For a given time, users can use the extended satellite orbit parameters to directly calculate satellite position, and the method is simple and high precision. While GLONASS selects the satellite position, velocity and the perturbation acceleration of the sun and moon as the broadcast ephemeris parameters, which are updated once per $0.5 \mathrm{~h}$. The GLONASS receiver can solve the differential equations of satellites perturbed motion by numerical integration method using the initial value of satellite motion state vector and to get the satellite position at any moment.

Because of perturbation model simplification and approximation, the satellite position calculation error can reach $0.57 \mathrm{~m}^{[1]}$. Based on analyzing the model of GLONASS broadcast ephemeris parameters user algorithm, a new GLONASS broadcast ephemeris parameters fitting model is established to fit the broadcast ephemeris parameters. The simulation shows that the average URE can be improved by nearly 2 orders of magnitude with the broadcast ephemeris parameters fitted. In addition, the effectiveness of the fitting method is verified by the open source RTKLIB tools.

\section{Broadcast Ephemeris Parameters User Algorithm}

GLONASS broadcast ephemeris parameters include ephemeris reference time, satellite position, velocity, perturbation acceleration of the sun and moon in PZ-90. Within period of validity of GLONASS broadcast ephemeris parameters, the simplified calculation algorithm of satellite position and velocity in PZ-90 is shown as follows.

$\frac{d x}{d t}=v_{x}=\dot{x}, \frac{d y}{d t}=v_{y}=\dot{y}, \frac{d z}{d t}=v_{z}=\dot{z}$ 
$\frac{d v_{x}}{d t}=-\frac{\mu}{r^{3}} x-J \mathrm{a} x+\omega^{2} x+2 \omega v_{y}+\ddot{x}_{s}, \frac{d v_{y}}{d t}=-\frac{\mu}{r^{3}} y-J \mathrm{a} y+\omega^{2} y-2 \omega v_{x}+\ddot{y}_{s}, \frac{d v_{z}}{d t}=-\frac{\mu}{r^{3}} z-J(2+\mathrm{a}) x+\ddot{z}_{s}$

Here $\mathrm{a}=1-5 z^{2} / r^{2}$. The other parameters can reference to the GLONASS ICD. It is necessary to state that there are two mistakes about formula 2 in GLONASS $\mathrm{ICD}^{[2]}$.

The formula 1 is the two order implicit ordinary differential equations. It is solved by four order Runge-Kutta numerical integration algorithm to obtain the satellite position and velocity at specified time in PZ-90 ${ }^{[2]}$. Within solving the differential equation, the step length is the key problem. Experiments show that the step length of $0.1 \mathrm{~s}, 1 \mathrm{~s}$ and $30 \mathrm{~s}$ have similar accuracy ${ }^{[3]}$.

\section{Broadcast Ephemeris Parameters Fitting Algorithm}

At present, the positioning accuracy of GLONASS is improved significantly from $25 \mathrm{~m}$ in 2006 to $7 \mathrm{~m}$ in $2010^{[4]}$. The practical measurement results show that the GLONASS average positioning accuracy can reach $3.059 \mathrm{~m}$ on the horizontal direction and $0.585 \mathrm{~m}$ on elevation direction in parts of East $\mathrm{Asia}^{[5]}$.The average positioning accuracy of north, east and elevation direction in site-centric coordinate system are $2.1 \mathrm{~m}, 2.1 \mathrm{~m}$ and $6.0 \mathrm{~m}$ respectively ${ }^{[6]}$. Among them, the cumulative errors and signal-in-space range errors caused by GLONASS broadcast ephemeris is $0.77 \mathrm{~m}^{[2]}$ and $0.4 \mathrm{~m}^{[7]}$ respectively. In order to improve the average prediction accuracy of GLONASS broadcast ephemeris within period of validity, a new broadcast ephemeris fitting model is established to improve the prediction accuracy of GLONASS broadcast ephemeris. The broadcast ephemeris parameters fitted contain all the perturbation factor of satellite orbit. Therefore, the broadcast ephemeris fitted can predict the satellite position with a high accuracy.

According to GLONASS broadcast ephemeris user algorithm, the observation equation of nonlinear model and estimated parameters can be get as follows:

$$
X=\left(t_{b}, x, y, z, v_{x}, v_{y}, v_{z}, a_{x}, a_{y}, a_{z}\right)^{T}, Y=f\left(X^{T}, t\right)+\eta
$$

Among them, $\mathrm{f}$ is the function of $X, x, y, z, v_{x}, v_{y}, v_{z}, a_{x}, a_{y}, a_{z}$ are estimated parameters, $\eta$ is the random error matrix, $Y$ contains $m(m \geq 9)$ observations. Because the equation established based on user observation equation is nonlinear, the process that the broadcast ephemeris parameters is fitted is the least-squares estimation problem of nonlinear systems, which need to be linear and iterative method to solve nonlinear equations.

According to the formula 3, supposed that there is an initial estimation vector $\hat{X}_{0}$ of vector $X$, and the linear equation based on parameter vector $\hat{X}_{0}$ can be expressed as follows:

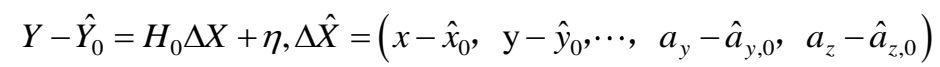

$\hat{Y}_{0}$ calculated using $\hat{X}_{0}$ by formula 3 is the estimation values observed data, $\Delta \hat{X}$ is the estimated parameter vector of $\Delta X . H_{0}$ is the Jacobi matrix which can be calculated as follows:

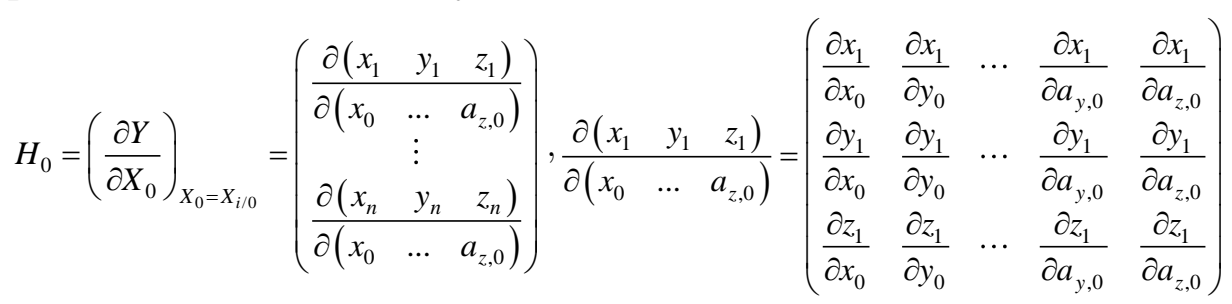

According to the principle of least square parameter estimation, the estimated vector value $\Delta \hat{X}$ in formula 4 can be expressed as:

$$
\begin{aligned}
& \Delta \hat{X}=X-\hat{X}_{0}=\left(H_{0}^{T} P^{-1} H_{0}\right)^{-1} H_{0}^{T} P^{-1}\left(Y-\hat{Y}_{0}\right) \\
& P=E\left(\eta \eta^{T}\right) \text { is considered as random error vector and } P \text { is the unit matrix. }
\end{aligned}
$$

Actually, the convergence condition of iterative process is set as follows:

$\left|\sigma_{i+1}-\sigma_{i}\right| / \sigma_{i}<\delta_{1}$ or The iteration number $<N$ 
$\delta_{1}$ and $\delta_{2}$ are set very small value according to the ephemeris fitting accuracy $\left(\delta_{1}=10^{-2}\right)$. $\mathrm{N}$ is the maximum number of iterations (Generally $N=30 \sim 50$ ), and $\sigma_{i}=\sqrt{v^{T} v /(m-15)}$ is the unit weight variance in the iterative of $i$.

As the GLONASS broadcast ephemeris parameters user algorithm is numerical integral algorithm, the function $f$ has no explicit expression and its partial derivative is very complex, which can't be expressed by conventional methods. Therefore, this paper presents a new calculation method using the difference quotient approximation instead of strict derivative calculation ${ }^{[8]}$. The difference quotient approximation principle can be show below:

$\partial Y / \partial x \approx\left(Y\left(x+\delta, y, \cdots, a_{y}, a_{z}\right)-Y\left(x, y, \cdots, a_{y}, a_{z}\right)\right) / \delta$

The parameter $\delta$ is small value (Generally $\delta=0.5 \sim 5$ ).

\section{Broadcast Ephemeris Parameters Fitting Algorithm}

Simulation. In order to verify the prediction accuracy of broadcast ephemeris fitted, the reference satellite precise orbit data is calculated by STK (Satellite Tool Kit), including earth non spherical gravitational perturbation, lunisolar perturbation, solar radiation perturbation and atmospheric drag perturbation. A corresponding satellite motion state is selected as the traditional broadcast ephemeris, Meanwhile, the series broadcast ephemeris parameters which are fitted out using the data in the half hour based on the broadcast ephemeris fitting method mentioned above. According to the two group broadcast ephemeris, their extrapolating accuracy of satellite position and speed can be calculated respectively using the GLONASS broadcast ephemeris parameters user algorithm.

The user ranging error (URE) is used to evaluate the forecast accuracy indexes of GLONASS broadcast ephemeris, which can be described as follows ${ }^{[9]}$ :

$$
U R E=\sqrt{R_{e r r}^{2}+0.0192 \times\left(\mathrm{T}_{e r r}^{2}+\mathrm{N}_{e r r}^{2}\right)}
$$

$R_{e r r}$ is the satellite radial error, $T_{e r r}$ is the satellite along track error, $N_{e r r}$ is the satellite normal error.

According to the simulation, the position forecast accuracy of fitting broadcast ephemeris is $0.0057 \mathrm{~m}$ which is two orders higher than traditional broadcast ephemeris of $0.3009 \mathrm{~m}$ in URE.

Positioning Precision. A GLONASS simulation system is constructed to simulate and generate observation data and broadcast ephemeris parameters. The observation data is simulated by multi-STK orbit data of satellites and the reference receiver position. Meanwhile the fitting ephemeris parameters and traditional ephemeris parameters files are generated respectively. Finally the observation data files and ephemeris parameters files are verified by RTKLIB tools. The RTKLIB tools is a free open source software for GNSS positioning, which can be used in GNSS standard and precise position $^{[15]}$.

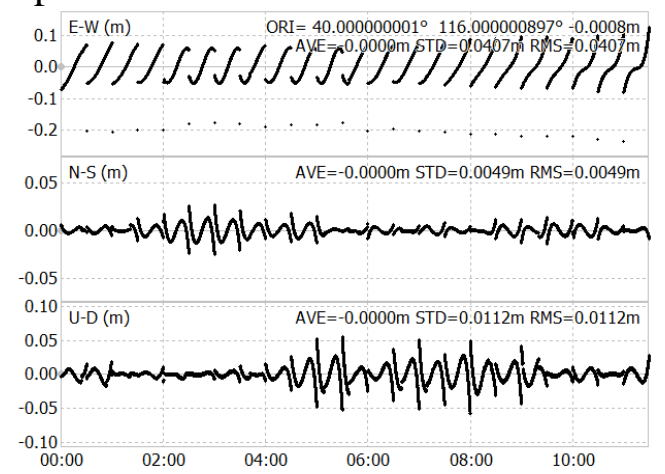

Fig 1. The positioning error curve of fitting broadcast ephemeris in $\mathrm{E} / \mathrm{N} / \mathrm{U}$

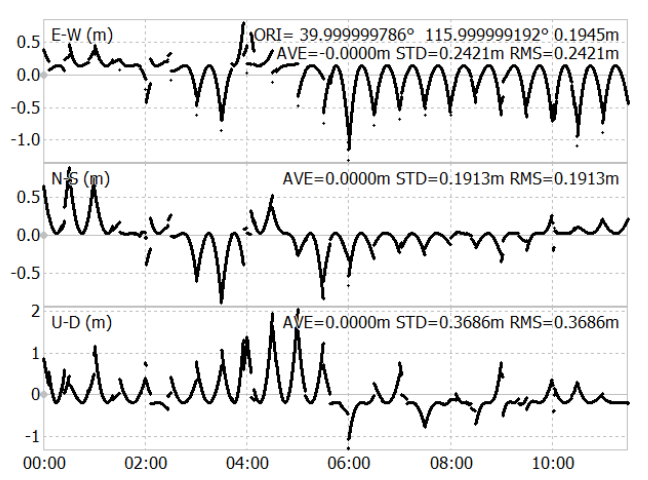

Fig 2 the positioning error curve of traditional. broadcast ephemeris in $\mathrm{E} / \mathrm{N} / \mathrm{U}$

In that simulation system, the simulation continuance time is set to an orbital period (11.5h). The broadcast ephemeris fitting interval is $0.5 \mathrm{~h}$, the broadcast ephemeris reference time is the 15 th $\mathrm{min}$ and 45 th min of every hour especially. 
Fig 1 and fig 2 give out respectively the positioning results using the two ephemeris parameters with the same observation data by RTKLIB RTKPOST tools. In the figures, the positioning accuracy of fitting ephemeris parameters in the $\mathrm{E} / \mathrm{N} / \mathrm{U}$ axis direction is better than traditional ephemeris parameters. The average positioning accuracy of fitting broadcast ephemeris parameters in the E/N/U axis direction are $0.0407 \mathrm{~m}, 0.0049 \mathrm{~m}$ and $0.0112 \mathrm{~m}$ respectively, and the average positioning accuracy of traditional broadcast ephemeris parameters in the E/N/U axis direction are $0.2421 \mathrm{~m}, 0.1913 \mathrm{~m}$ and $0.3686 \mathrm{~m}$. In addition, the fitting ephemeris parameters positioning error in the E/N/U axis direction changes more regularity and small fluctuation than the traditional ephemeris parameters. By comparison, the fitting broadcast ephemeris parameters has certain advantages and can improve the user position accuracy greatly.

\section{Conclusion}

According to the characteristics of the design of GLONASS broadcast ephemeris parameters and its user algorithm, the satellite orbit prediction accuracy of traditional broadcast ephemeris exists error accumulation. Aiming at this problem, a new GLONASS broadcast ephemeris fitting algorithm is proposed. The simulation results show that compared to the traditional broadcast ephemeris, satellite orbit average prediction accuracy using the fitting broadcast ephemeris is greatly improved. At the same time, a simulation system is constructed and the observation data file and two broadcast ephemeris files are generated by standard RINEX 3 format. The two broadcast ephemeris are verified using the Japan's open source RTKLIB tools from positioning point of view. The high accuracy and applicability of fitting broadcast ephemeris are proved.

\section{References}

[1] Hu Songjie. Research on the Broadcast Ephemeris Parameters of GPS and GLONASS [J], Journal of Spacecraft TT\&C Technology,2005, 3(24):37-42.

[2] Interface Control Document, Edition 5.1, MOSCOW, 2008.

[3] Li Zhi, Zhang Ruoyu. Orbit Computation and Data Fusion of GPS/GLONASS Integrated Navigation System[J], Journal of Institute of Command and Technology, 1999, 10(6): 19-22.

[4]Ekaterina Oleynik, Sergey Revnivykh. GLONA -SS Status and Modernization[C]. Civil GPS Service Interface Committee Portland, Oregon, 2011.

[5]Sakai, Takeyasu, Yamada, el at. "GPS /GLONASS Multi-Constellation SBAS Trial and Preliminary Results for East-Asia Region," Proceedings of the 25th International Technical Meeting of The Satellite Division of the Institute of Navigation, Nashville, TN, September 2012, pp. 854-866.

[6]Sergey Revnivykh, Ekaterina Oleynik. GLON -ASS Performance Monitoring[C]. ION GNSS 2012, GLONASS Workshop Nashville, Tennessee, 2012.

[7] Revnivykh, Sergey, "GLONASS Ground Control Segment: Orbit, Clock, Time Scale and Geodesy Definition," Proceedings of the 25th International Technical Meeting of The Satellite Division of the Institute of Navigation, Nashville, TN, September 2012, pp. 3931-3949.

[8]Xing Zhicheng, Wang Xiexian, Method of Calculating Numerical Derivative of Jacobin Matrix for Fitting Algorithm of GPS Broadcast Ephemeris Parameters[J], GNSS World of China, 2012,37(1):28-31.

[9]Ananda M P, Berstein H, CunningHam K E, et al. Global Positioning System(GPS) Autonomous Navigation[C], ESA SP-425, The 4th ESA International Conference on Spacecraft Guidance, Navigation Control System, ESTEC, Netherlands, October 18-21,1991. 